\title{
Augmented Reality, Cyber-Physical Systems and Robotics: Nice to Have or a Program with Future?
}

\author{
Hubertus Feußnera, b Beate Rauc

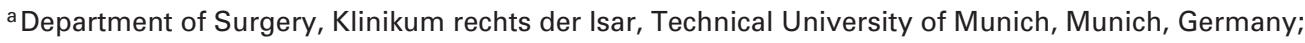 \\ ${ }^{b}$ Working Group for Minimally Invasive Interdisciplinary Therapeutical Intervention, Klinikum rechts der Isar, Technical University \\ of Munich, Munich, Germany; \\ ${ }^{\mathrm{c}}$ Department of Surgery, Campus Virchow-Klinikum and Charité Campus Mitte, Charité - Universitätsmedizin Berlin, Berlin, Germany
}

Striving for the continuous improvement of patient care is an ethical obligation for all stakeholders in medical care but in particular for the surgeon. Accordingly, any technical innovations with the potential to ameliorate our abilities to fight disease more effectively than today have to be observed attentively. Hopefully, they might offer the chance of further improving our treatment options.

However, surgery requires a continuous development per se and even beyond ethical reasons, since the general environment is highly competitive. Many formerly surgical indications have become the domain either of conservative treatment (like peptic ulcer disease) or of interventional gastroenterology or radiology.

If surgery strives for retaining its role in medical care giving, it must be capable of coping with the challenges of the 'magic triangle': reduction of trauma and expenses and, simultaneously, the improvement of outcome. In short: We need even better and more economical approaches.

Further progress in surgery depends on the use of innovative technologies, and we should carefully look at emerging new methods as to whether they might be helpful for surgery. An additional obligation is to evaluate new products on the market and to assess their true value according to best scientific practice as soon as possible. If a new procedure/device etc. is really advantageous, patients have a right to obtain it as soon as possible. On the contrary, new approaches should not be integrated into routine clinical care if their superiority cannot be proven.

This is the background of this issue of Visceral Medicine with the topic 'Augmented Reality, Cyber-Physical Systems and Robotic Surgery'.

The range is rather broad. Robotics are part of the topic; however, they are only one aspect of the new technologies in surgery. Most probably, other promising innovations - admittedly less spectacular than robots - will have an even more significant impact on surgery. We tried to denote them by the terms 'augmented reality' and 'cyber-physical systems'.

\section{KARGER}

(c) 2018 S. Karger GmbH, Freiburg
Whereas the reader is familiar with the meaning of 'virtual reality' and even 'augmented reality' (e.g. a combined presentation of the actual anatomical presentation of the actual anatomical view with preoperative imaging on the same screen), the general idea behind the term 'cyber-physical systems' needs, perhaps, some explanation. Cyber-physical systems are a network of interacting hardware and software components working together with the support of computational intelligence which includes the capability of communication with the human user. At first glance, this concept, which is the theoretical basis of the 'industry 4.0 ' initiative, appears to be far away from surgery, but the contrary is true. The surgical operating room is becoming an increasingly technicized workplace which urgently needs a better organization, an improved safety level, and a reduction of the workload of the surgical team. An increasing number of workgroups all over the world is currently active in order to realize the idea of 'surgery 4.0', which can almost be considered as a synonym for 'cyber-physical systems in surgery'.

At the moment, however, the surgical community is still focused on the question of 'robotics' in surgery. Just to mention it: The term 'robot' is completely inadequate to describe a da Vinci or any other system. What they really are is defined as 'telemanipulation systems'.

Nonetheless, 'robots' are currently at the top of the ranking list in general surgery, and accordingly, as many as three contributions of this issue of Visceral Medicine are devoted to da Vinci applications in surgery: Biebl et al. [1] outweigh the aspects of robotic versus laparoscopic procedures in upper gastrointestinal surgery. Baukloh et al. [2] try to do the same for lower gastrointestinal surgery. Stiegler and Schemmer [3] analyze the question whether robot-assisted transplantation surgery is still a vision or already reality. If these contributions are read carefully, at least two aspects become evident. Firstly, the authors cannot conceal a certain sympathy for the use of telemanipulation systems in surgery, and, sec-

Prof. Dr. Hubertus Feußner 
ondly, their meticulous analysis of the literature is unable to prove a clear superiority of the use of telemanipulation systems when compared to the laparoscopic approach. As the guest editors of this issue, we dare to point out that the successful use of a 'robotic' system is not a value of its own right in surgery!

After so many papers and even prospective randomized trials and meta-analyses, there is almost no doubt any longer that most surgeries can be performed successfully by telemanipulation systems. The question nowadays, however, is whether they can be really recommended as superior? Certainly not! It is the natural reflex of the protagonists (and not to forget the providers) to demand new trials. Taking the flood of information into account which is available today (about 18 years after the introduction of this type of devices), the question might be warranted if another series of randomized trials is really suitable to identify niches for the 'robot'? It reminds of the alchemists of ancient times who asked for yet another new experiment to finally achieve the goal of transforming lead into gold.

It could just now be the time to conclude that our current generation of 'robots' is not (yet) superior to conventional laparoscopic surgery, if all pros and cons are considered. One of the major 'cons' are the expenses. Costs might decline if the monopoly situation ceases with new, competitive systems. Stephan et al. [4] introduce a new telemanipulation system which seems to be an alternative and valuable option to the currently leading system.

A 'pro' in favor of the telemanipulation system is excellent visualization. Ohmura et al. [5] demonstrate that this aspect can easily be provided by a camera navigation system. In this regard, camera guidance systems which are by far less expensive than complete telemanipulation systems could become valuable.

As an outlook, the paper of $\mathrm{Li}$ and Chiu [6] deals with the topic of (endoluminal) robotic endoscopy. Though it is still in the beginning, telemanipulation systems for endoluminal endoscopy will certainly be available in the long run.
Good visual information is an essential pillar of each type of surgery. Wilhelm et al. [7] present the various technologies providing augmented reality and highlight some clinical applications. Beyond augmented reality, enhanced visualization also includes intraoperative tissue differentiation which enables us to perform an even more precise, individualized and patient-friendly surgery.

Implicitly, this paper on enhanced visualization also emphasizes the central importance of cyber-physical systems. Without smart cooperation of computers and devices (sensors, image processors, operating room table, machines for artificial ventilation, etc.) the problem of registration (alignment of the actual operative situation with preoperative imaging) would never work reliably. An autonomous communication and cooperation of numerous technical systems is an essential precondition of augmented reality.

What is more, the conflicting opinions and sometimes subjective, differing views on the aspects of this special topic are given a forum in the 'Interdisciplinary Discussion' chaired by Kirschniak [8].

We are firmly convinced that a metamorphosis of currently available telemanipulation devices into cyber-physical systems would initiate, finally, the real breakthrough of robots in surgery. This transformation as a disruptive step would offer to surgery what it really needs: collaborative, situation-aware assistance systems.

In conclusion, advanced technologies in surgery are by far more than a 'nice to have'. They are the Achilles heel in order to achieve a new type of surgery which can be denominated as evidence-based or cognitive surgery. This cognitive surgery or 'surgery 4.0 ' is the key to preserve the leading role of surgery in interventional medicine.

\section{Disclosure Statement}

The authors do not have a conflict of interest to declare.

\section{References}

1 Biebl M, Andreou A, Chopra S, Denecke C, Pratschke J: Upper gastrointestinal surgery: robotic surgery versus laparoscopic procedures for esophageal malignancy. Visc Med 2018;34:DOI: 10.1159/000487011.

2 Baukloh JK, Perez DR, Reeh M, Biebl M, Izbicki JR, Pratschke J, Aigner F: Lower gastrointestinal surgery: robotic surgery versus laparoscopic procedures. Visc Med 2018;34:DOI: 10.1159/000486008.

3 Stiegler P, Schemmer P: Robot-assisted transplant surgery - vision or reality? A comprehensive review. Visc Med 2018;34:DOI: 10.1159/000485686.
4 Stephan D, Sälzer H, Willeke F: First experiences with the new Senhance ${ }^{\circledR}$ telerobotic system in visceral surgery. Visc Med 2018;34:DOI: 10.1159/000486111.

5 Ohmura Y, Nakagawa M, Suzuki H, Kotani K, Teramoto A: Feasibility and usefulness of a joystick-guided robotic scope holder (Soloassist) in laparoscopic surgery. Visc Med 2018;34:DOI: 10.1159/000485524.

6 Li Z, Chiu PW-Y: Robotic endoscopy. Visc Med 2018; 34:DOI: $10.1159 / 000486121$.
7 Wilhelm D, Vogel T, Ostler D, Marahrens N, Kohn N Koller S, Friess H, Kranzfelder: Enhanced visualization: from intraoperative tissue differentiation to augmented reality. Visc Med 2018;34:DOI: 10.1159/000485940.

8 Kirschniak A, Egberts J-H, Granderath F-A, Mees ST, Mille M, Müller BP, Rautio T, Röth AA: Augmented reality, cyber-physical systems and robotic surgery: nice to have or a program with future? (Interdisciplinary Discussion). Visc Med 2018;34:DOI: 10.1159/000487209. 